EPJ Web of Conferences 35, 04003 (2012)

DOI: $10.1051 /$ epjconf/20123504003

(C) Owned by the authors, published by EDP Sciences, 2012

\title{
Atomic Radiation in Nuclear Decay
}

\author{
B.Q. Lee $^{1, a}$, T. Kibédi ${ }^{1}$, A.E. Stuchbery ${ }^{1}$, and K.A. Robertson ${ }^{1}$
}

Department of Nuclear Physics, Research School of Physics and Engineering, The Australian National University, Canberra, ACT 0200, Australia

\begin{abstract}
Auger electrons emitted in nuclear decay offer a unique tool to kill cancer cells at the scale of a DNA molecule. Over the last forty years many aspects of this promising therapeutic tool have been explored, however it is still not in the phase of large scale clinical trials. In this paper we review the physical processes of Auger emission in nuclear decay and present a new model being developed to evaluate the energy spectrum of Auger electrons, and hence overcome the limitations of existing computations.
\end{abstract}

\section{Introduction}

Unstable atomic nuclei release excess energy through various radioactive decay processes by emitting radiation in the form of particles (neutrons, alpha, beta particles) or electromagnetic radiation (gamma-ray photons). Most of the applications using nuclear isotopes are based on the fact that the interaction of the radiations passing through material will depend on their type (photons, neutral or charged particles) and the transferred energy. Most radioisotopes used in clinical therapy emit $\beta$ particles, which are ionizing radiations. The biological effect is often characterized by Relative Biological Effectiveness (RBE) which is related to Linear Energy Transfer, LET. LET is expressed in units of $\mathrm{keV} / \mu \mathrm{m}$, which is a measure of the energy deposited along the particle track. A new class of radionuclides [1], including ${ }^{149} \mathrm{~Tb},{ }^{211} \mathrm{At},{ }^{211} \mathrm{Po},{ }^{213} \mathrm{Bi},{ }^{223} \mathrm{Ra}$, ${ }^{225} \mathrm{Ac},{ }^{226} \mathrm{Th},{ }^{227} \mathrm{Ac}$, and ${ }^{230} \mathrm{U}$, which emit $\alpha$ particles have been considered for therapy. The LET for most therapeutic $\alpha$ emitters ranges from 25 to $230 \mathrm{keV} / \mu \mathrm{m}$. On the other hand, electrons and positrons emitted in nuclear $\beta$ decay, and in the internal conversion processes, referred to here as $\beta$ particles, have kinetic energies ranging from tens of $\mathrm{keV}$ to several MeV and their LET is much lower, typically $\sim 0.2 \mathrm{keV} / \mu \mathrm{m}$.

A third type of ionizing radiation is Auger electrons [2], named after the French physicist Pierre Victor Auger. When an inner-shell electron is removed from an atom, the vacancy will be filled by an electron from the outer shells and the excess energy will be released as an X-ray photon, or by the emission of an Auger electron. Referred to as atomic radiations, $\mathrm{X}$-ray and Auger electron emission are competing processes. The atomic transition rate and the energy of emitted X-rays and/or Auger electrons depend on the atomic number, the electron shells involved, and the electron configuration of the atom. The full relaxation of the inner-shell vacancy is a multi step process, resulting in a cascade of atomic radiations. The energy of atomic radiation is typically in the range from a few eV to $100 \mathrm{keV}$. Due to their short range (nm to $\mu \mathrm{m}$ ), Auger electrons with relatively low energies can have a much higher LET. For

\footnotetext{
a e-mail: u4659773@anu.edu.au
}

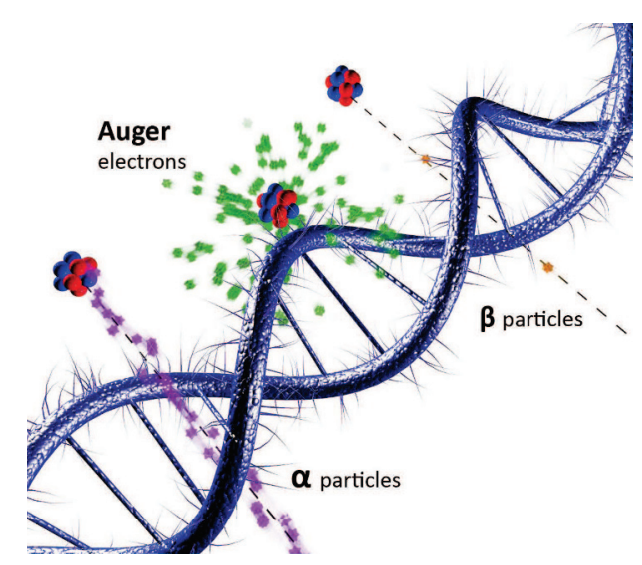

Fig. 1. Interactions of ionizing radiations on the scale of DNA. The green, yellow and purple dots represent individual ionization events and hence their corresponding LET. (Courtesy of Thomas Tunningley, ANU)

example, for electron energies below $1 \mathrm{keV}$ the LET peaks at around $26 \mathrm{keV} / \mu \mathrm{m} \mathrm{[3].} \mathrm{In} \mathrm{comparison} \mathrm{to} \alpha$ or $\beta$ particles, Auger electrons have a much shorter range in material, which make them ideal tools for targeted radiation therapy. Fig. 1 shows a pictorial comparison of the interaction sites for these three types of ionizing radiation. Other than these three ionizing radiation, many medical applications also use low LET ionizing radiation such as gamma rays and x-rays emitted from radioisotopes.

In this paper we will only focus on the physical processes required to evaluate the Auger emission in nuclear decay. We start our discussion with an overview of the current knowledge; then we propose a new approach to overcome the limitations of the current computations used for low-energy Auger emission from medical isotopes.

\section{Radioactive decay processes}

When a vacancy is created in an inner electron shell, the residual atom is left in an excited state. Such a vacancy can be created by nuclear decays such as electron capture (EC), or internal conversion electron (CE) processes. 


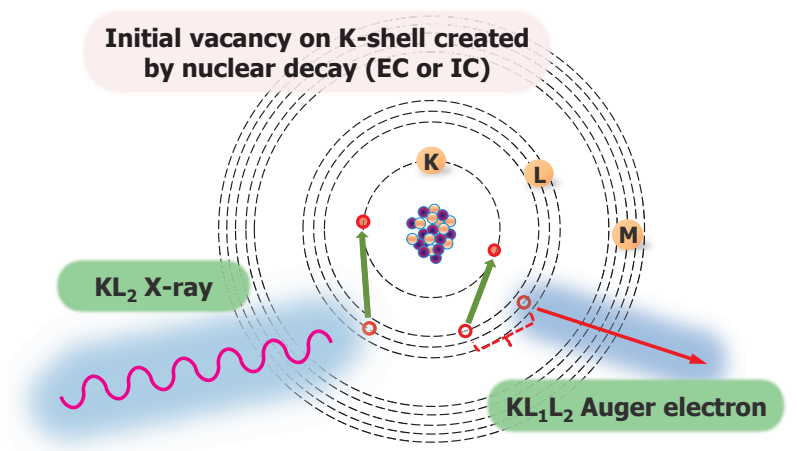

Fig. 2. Relaxation of a vacancy in the $K$ shell by X-ray and Auger emission.

Typical atomic events involving the $K$-shell are shown in Fig. 2.

In electron capture the nucleus decays by absorbing an atomic electron and emitting a neutrino:

$$
(Z+1, A)+e \rightarrow(Z, A)+v_{e}
$$

The condition for electron capture decay is

$$
E_{v}=Q^{+}-E_{i}-E_{X}>0,
$$

where $Q^{+}$is the energy difference in atomic masses between parent and daughter ground states, $E_{i}$ is the energy of the final nuclear state in the daughter nucleus, and $E_{X}$ is the binding energy of the captured electron, $X$. The released energy, $E_{v}$ will be shared by the emitted neutrino and, if applicable, the Bremsstrahlung photon or shaking electron. Comprehensive compilation of the relevant electron capture probability ratios for the $K, L, M, N$ and $O$ shells were presented by Schönfeld [4].

Nuclei undergoing electromagnetic decays will emit $\gamma$-rays, internal conversion electrons, or if the transition energy is higher than twice the electron rest mass, electronpositron pairs. In the internal conversion process an atomic electron is ejected from one of the atomic shells. The electron conversion coefficient is defined as the ratio of the probabilities of the emission of atomic electrons from shell $X\left(P_{X}\right)$ to the emission of $\gamma$-rays $\left(P_{\gamma}\right)$ :

$$
\alpha_{X}=\frac{P_{X}}{P_{\gamma}} .
$$

Theoretical internal conversion electron emission rates can be obtained from [5].

\section{$3 \mathrm{X}$-rays and Auger electrons}

It is customary to assume that the radioactive atom initially is in the neutral, ground state electronic configuration. Immediately after an electron capture or internal conversion event, the atom will be excited. In 1923, S. Rosseland [6] postulated that the atom relaxes via both radiative and non-radiative processes. Radiative processes will involve the emission of $\mathrm{X}$-rays with characteristic energies as the atomic electrons are reorganized to fill the vacancy. In X-ray emission, an electron in an outer shell, Y, makes a transition to a vacancy in the inner shell, $\mathrm{X}$, and the emitted energy of the $\mathrm{X}$-ray is:

$$
E_{X Y}=E_{B E, X}-E_{B E, Y},
$$

where $E_{B E, X}$ and $E_{B E, Y}$ are the binding energies of the atomic shells involved. The fluorescence yield, $\omega_{X}$, is defined as the number of radiative (X-ray) transitions per vacancy in any shell or subshell $X$. Considering all possible shells, subshells, $Y$, involved in filling the vacancy on the $K$ shell ( $X$ is equal to $K$-shell), the $\mathrm{X}$-ray yield, $Y_{K Y}$ can be expressed as:

$$
Y_{K Y}=f_{K} \omega_{K} N_{K Y},
$$

where $f_{K}$ is the number of primary vacancies on the $K-$ shell, and $N_{K Y}$ is the relative intensity of various $\mathrm{X}$-ray transitions with $\sum N_{K Y}=1$.

Pierre Auger made the first confirmed experimental observation of the non-radiative process in 1925 [2]. Nonradiative processes similarly involve the redistribution of atomic electrons but result in the emission of an atomic electron (Auger electron). The Auger electron process $X Y Z$ involves three electron (sub-)shells. An electron in an outer shell, $Y$, makes a transition to the vacancy in an inner shell, $X$, and an electron in outer shell $Z$ is ejected. The energy of the Auger electron can be expressed as:

$$
E_{X Y Z}=E_{B E, X}-E_{B E, Y}-E_{Z}^{Y},
$$

where $E_{B E, X}$ and $E_{B E, Y}$ are the neutral atom binding energies for shell $X$ and $Y . E_{Z}^{Y}$ is the binding energy of an electron on the $Z$-shell when the atom is already ionized with a single vacancy on the atomic shell $Y$. This process will result in vacancies in both the $Y$ and $Z$ shells from a single initial vacancy in the $X$ shell. Similarly to Eqn. 5 the Auger electron yield can be expressed as:

$$
Y_{K Y Z}=f_{K}\left(1-\omega_{K}\right) N_{K Y Z},
$$

where $N_{K Y Z}$ is the relative intensity of various Auger transitions with $\sum \sum N_{K Y Z}=1$. The sums are over all energetically possible $Y$ and all possible $Z$ with binding energies $E_{B E, Y} \geq E_{B E, Z}$.

\section{Vacancy propagation}

The rearrangement of the atomic structure will continue until all primary, secondary and subsequent vacancies are filled by the emission of X-rays and Auger electrons, or until no more transitions are energetically possible. In the latter case, the vacancy has reached the valence shell.

The full relaxation of the initial vacancy created in the nuclear event (section 2) is a multi step process. While the fundamental physical picture of the individual atomic transitions remains similar to the one described above, the atomic structure will continuously change. This change will effect both the atomic binding energies and transition rates.

Table 1 compares the various calculated Auger electron yields of radioisotopes of medical importance. These include ${ }^{99 m} \mathrm{Tc},{ }^{111} \mathrm{In},{ }^{123,125} \mathrm{I}$ and ${ }^{201} \mathrm{Tl}$. The table contains six calculations, which follow two fundamentally different approaches: deterministic and Monte-Carlo. 
In "deterministic approach", the contributions from filling each vacancy are computed using closed formulae, similar to Eqns. 5 and 7. It was used by the RAdiation Dose Assessment Resource (RADAR) [7,8], the Decay Data Evaluation Project (DDEP) [9] and Eckerman $\&$ Endo [10]. This approach is quite reasonable and simple for transitions involving vacancies on the $K$ and $L$ shells. However a more realistic description must include the outer shells and hence requires that a very large number of transitions be considered. In general, the accuracy of these "deterministic predictions" largely depends on the inclusion of outer shells.

An alternative approach is to base the calculations on "Monte Carlo" (MC) techniques, which prove to be better suited to the inclusion of all possible paths in the relaxation process. Such simulations begin with the selection of the nuclear decay process and the consequent creation of the initial vacancy. During the propagation of the initial vacancy, the next transition is randomly selected from all available atomic transitions, using the transition rates as weighting factors. Table 1 includes results from Howell [11], Stepanek [12] and a very recent calculation by Pomplun [13]. The Monte Carlo approach allows the incorporation of all atomic shells.

Common in both approaches is the necessity to know all relevant transition energies and transition rates. All 6 calculations listed in Table 1 use transition rates from existing tabulations based on a combination of experimental data, systematics (obtained by interpolation and extrapolation), as well as theoretical calculations, which often used different assumptions, wave functions, etc. Most of the data presented in these compilations are for cases when there is a single vacancy on one of the atomic shells. In an effort to compensate for this limitation, the calculations presented in Table 1 have employed various corrections. One of these is the so-called Krause-Carlson correction [14], which takes into account the effect of multiple vacancies on a shell accumulated in the course of the relaxation process. Most of these calculations neglect the shakeup and shakeoff effect, which might be significant for transition rates when a vacancy is created on the outermost shells [15].

The transition energies are usually derived from atomic binding energies. As for the transition rates, the atomic binding energies are also affected by changes in the atomic configuration occurring during the relaxation process. Some of the calculations listed in Table 1 simply use neutral atom binding energies (RADAR, Eckerman \& Endo and Howell) or semi-empirical values from Larkins [16] (DDEP). Others use the $Z / Z+1$ rule [17] (Howell) to estimate the Auger electron energies. Only the two most recent Monte Carlo approaches (Stepanek [12] and Pomplun [13]) use theoretical values obtained from relativistic Dirac-Fock calculations.

In summary, existing computations of Auger electron spectra are far from complete. Most of them are based on transition rates and transition energies obtained for single vacancies.

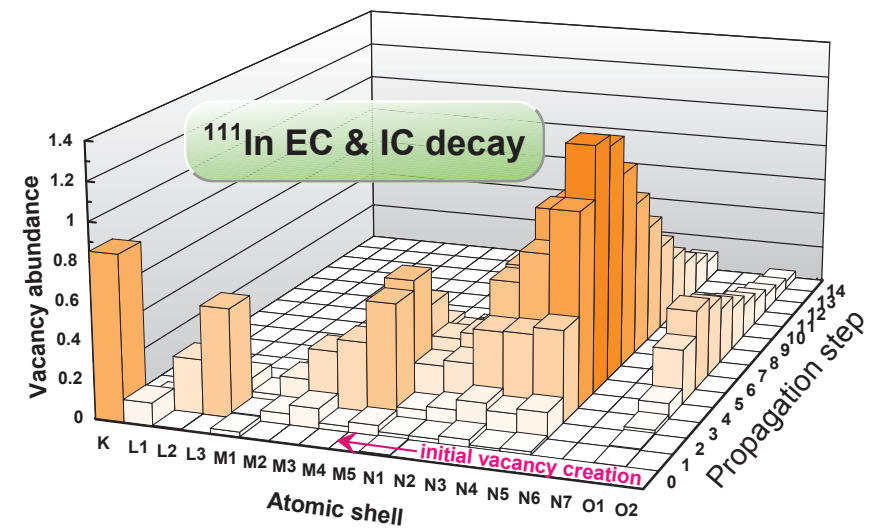

Fig. 3. Vacancies created during the relaxation process in ${ }^{111}$ In.

\section{New ab initio calculations of Auger transition rates - A pilot study}

The starting point to fully explore the potential of the targeted Auger electron based therapy is an accurate description of the relevant atomic radiation spectrum from the decaying radioisotopes. Recognizing the lack of a consistent theoretical model, the August 2011 IAEA special meeting on Intermediate-term Nuclear Data Needs for Medical Applications [18] concluded that: "A comprehensive calculational route also needs to be developed to determine the energies and emission probabilities of the low-energy $X$-rays and Auger electrons to a higher degree of detail and consistency than is available at present." To improve the understanding of the atomic radiation spectra in nuclear decay a new approach is required, which should use new theoretical transition energies and rates. In addressing this need we propose to adopt the following protocol for a new Monte Carlo approach:

Nuclear structure data will be extracted from the Evaluated Nuclear Structure File (ENSDF) [19]. ENSDF is maintained regularly and this will ensure the use of the most up-to-date information to evaluate the nuclear event.

Electron capture rates will be taken from the Schönfeld (1995) compilation [4]. Internal conversion coefficients (ICC) will be taken from BrIcc [5]. The ICC values in that tablulation were calculated using relativistic Dirac-Fock wave functions.

Auger and X-ray transition energies and rates will be calculated using the most recent version of the General Purpose Relativistic Atomic Structure program, GRASP2K [20] and the Relativistic Atomic Transition and Ionization Properties, RATIP [21] codes. The RATIP program package was developed in the late 90s for the calculation of atomic transition and ionization properties for charged ions [22], similar to those expected during the vacancy propagation process. Calculations will be carried out at every propagation step for the actual atomic configuration of the ionized atom.

The vacancy creation and the atomic relaxation processes from EC decay and from internal conversion will be treated independently. In all practical cases IC takes place after the daughter atom is fully relaxed following an EC event, so internal conversion takes place in a neutral atom. 
Table 1. Calculated Auger electron yields for selected medical radioisotopes.

\begin{tabular}{lccccccc}
\hline & $\begin{array}{c}\operatorname{RADAR}^{a} \\
{[7,8]}\end{array}$ & $\begin{array}{c}\text { DDEP }^{a} \\
{[9]}\end{array}$ & $\begin{array}{c}\text { Eckerman \& Endo }^{a} \\
{[10]}\end{array}$ & $\begin{array}{c}\text { Howell }^{b} \\
{[11]}\end{array}$ & $\begin{array}{c}\text { Stepanek }^{b} \\
{[12]}\end{array}$ & $\begin{array}{c}\text { Pomplun }^{b} \\
{[13]}\end{array}$ & Present study $^{b}$ \\
\cline { 2 - 7 } & \multicolumn{7}{c}{ Auger electron yield per nuclear decay } \\
${ }^{99 m} \mathrm{Tc}(6.007 \mathrm{~h})$ & 0.122 & 0.13 & 4.363 & 4.0 & & 2.5 & 3.37 \\
${ }^{111} \mathrm{In}(2.805 \mathrm{~d})$ & 1.136 & 1.16 & 7.215 & 14.7 & 6.05 & & 5.75 \\
${ }^{123} \mathrm{I}(13.22 \mathrm{~h})$ & 1.064 & 1.08 & 13.71 & 14.9 & & 6.4 & \\
${ }^{125} \mathrm{I}(59.4 \mathrm{~d})$ & 1.77 & 1.78 & 23.0 & 24.9 & 15.3 & & \\
${ }^{201} \mathrm{Tl}(3.04 \mathrm{~d})$ & 0.773 & 0.614 & 20.9 & 36.9 & &
\end{tabular}

${ }^{b}$ Monte Carlo

This assumption is not valid in rare cases wherein the nuclear level half life of the daughter nucleus is much shorter than the time needed for the atom to fully relax. For example, a shift of $20 \pm 7 \mathrm{eV}$ of the $K$ conversion electron line of the $963 \mathrm{keV}$ transition in the electron capture decay of ${ }^{152} \mathrm{Eu}[23]$ is one of the few experimental observations of this rare scenario.

The $a b$ initio treatment of the propagation process including the random sampling of the available decay channels will ensure the realistic evaluation of the atomic spectra. In this approach the atom is assumed to be free, and any influence from the chemical environment or solid state effects is neglected. In our model the propagation of a particular vacancy will be terminated if there is no higher state energetically available, or if it has reached the valence shell. However the propagation of the event is not complete while there are any inner vacancies still left; the propagation of these vacancies will continue until all have reached the valence shell. In the proposed model it will be assumed that the vacancies on the valence shell(s) will remain unfilled throughout the entire relaxation process.

To explore the implications of this new approach, a pilot model was developed. This model follows the proposed approach, except that fixed atomic transition rates were taken from the EADL [24] data base. Transition energies were deduced from binding energies given by the RAINE Dirac-Fock code [25], and transitions with negative energies were excluded.

Representative numbers of the Auger electrons emitted in the decay of ${ }^{99 m} \mathrm{Tc}$ and ${ }^{111} \mathrm{In}$ is shown in Table 1.

Fig. 3 shows the abundance of atomic vacancies for each atomic shell during the atomic cascade. Vacancy creation from the nuclear decay occurs at step " $O$ " and events with up to 14 propagation steps are indicated. The plot was generated by evaluating 1,000,000 EC decays of ${ }^{111} \mathrm{In}$, one of the commonly used radioisotopes for nuclear imaging. More than $97.5 \%$ of the initial vacancies are from electron capture on the $K$ - and $L_{1}$-shells. Closer examination of the graph reveals how the vacancies "migrate" towards the outer shells. For most of the events, the created vacancies take 7 or 8 propagation steps to reach the outer shells. Beyond that number of propagation steps the vacancy abundance in Fig. 3 shows a decrease because events with more steps become increasingly unlikely. Some key features of the propagation process include: $(i)$ The highest abundances of the vacancies are on the last subshell of each major shell: $L_{3}, M_{5}$, and $N_{5}$. $\left(N_{6}\right.$ and $N_{7}$ are not occupied.) (ii) As the vacancies approach the outer shells ( $M$ and $N$ ) they are retained longer; i.e. they are more likely to survive for several propagation steps.

An important result of the pilot model is the calculated total yield of Auger electrons: ${ }^{99 m} \mathrm{Tc}$ : 3.37 ; and ${ }^{111} \mathrm{In}: 5.75$ electrons per radioactive decay of the parent atom. These results are generated from the simulations of one million nuclear decays to ensure the calculated yield converges. The main uncertainties in the evaluation process are coming from EADL data base and RAINE program. EADL data base does not specify the uncertainties in their calculations and theoretical neutral binding energies from RAINE diverge from empirical values by up to about $1 \%$. In accord with our assumption that valence-shell vacancies persist, these results are consistent with those of Pomplun [13]. We have therefore demonstrated that we can reproduce the previous Monte Carlo calculations for these isotopes.

\section{Conclusions}

There is continuing interest in medical applications of Auger electrons which accompany nuclear decay, particularly for the targeted treatment of cancer cells at the DNA scale. In most cases these applications are based on theoretical predictions of the emitted Auger and X-ray spectra. As it is evident from Table 1, there is a significant difference in the Auger yields reported in the literature over the last 20 years. Most of these differences can be attributed to the lack of detailed knowledge of the relevant atomic transition rates, most prominently in the outer $(M, N$, etc.) shells. Simplistic assumptions regarding the atomic configurations during vacancy propagation and the incomplete treatment of the effect of multiple vacancies also limit the validity of these predictions.

We are developing a new model using ab initio calculations based on the relativistic Dirac-Fock approach and Monte Carlo techniques, which has the potential to overcome these limitations. Pilot calculations for the isotopes ${ }^{99 m} \mathrm{Tc}$ and ${ }^{111} \mathrm{In}$, based on fixed transition rates from the EADL database [24], are in satisfactory agreement with previous computations. 


\section{References}

1. S. George, Advanced Drug Delivery Reviews 60, (2008) 1402

2. P. Auger, Journal de Physique et le Radium 6, (1925) 205

3. A.I. Kassis, International Journal of Radiation Biology 80, (2004) 789

4. E. Schönfeld, Appl. Radiat. Isot. 49, (1998) 1353

5. T. Kibédi et al, Nucl. Instr. and Meth. in Phys. Res. A 589, (2008) 202

6. R. Rosseland, Zeitschrift für Physik A Hadrons an Nuclei 14, (1923) 17

7. RAdiation Dose Assessment Resource (RADAR), http://www . doseinfo-radar . com/RADARHome .

html.

8. M.G. Stabin, L,C.Q.P. da Luz, Health. Phys. 83, (2002) 471

9. Decay Data Evaluation Project, http://www. nucleide.org/DDEP_WG/DDEPdata.htm, ${ }^{99 m}$ Tc: C. Morillon, M.M. Bé, A. Egorov (2012); ${ }^{111}$ In: V.P. Chechev (2006); ${ }^{123} \mathrm{I}$ : V. Chiste, M.M. Bé (2004); ${ }^{125} \mathrm{I}$ : V. Chiste, M.M. Bé (2010); ${ }^{201}$ Tl: E. Schönfeld, R. Dersch (2005)

10. K.F. Eckerman et al, VA, Reston, Society of Nuclear Medicine, (2007)

11. R.W. Howell, Medical Physics 19, (1992) 1371

12. J. Stepanek, Medical Physics 27, (2000) 1544

13. E. Pomplun, Int. J. of Radiation Biology 88, (2012) 108

14. M.O. Krause et al, Phys. Rev. 158, (1967) 18

15. T.A. Carlson et al, Phys. Rev. A 8, (1973) 2887

16. F.P. Larkins, At. Data and Nucl. Data Tabl. 20, (1977) 311

17. M.F. Chung et al, Surface Science 22, (1970) 479

18. Summary Report of the "Technical Meeting on Intermediate-term Nuclear Data Needs for Medical Applications: Cross Sections and Decay Data", INDC International Nuclear Data Committee, Eds. A.L. Nichols, S.M. Qaim, R.C. Noy, INDC(NDS)-0596, September 2011.

19. Evaluated Nuclear Structure Data File (ENSDF), NNDC, BNL http://wWw.nndc.bnl.gov/ensdf/ index.jsp

20. P. Jönsson et al, Comp. Phys. Comm. 177, (2007) 597

21. J. Nikkinen et al, Comp. Phys. Comm. 175, (2006) 348

22. M. Patanen et al, Physical Review A 83, (2011) 053408

23. V.V. Bulgakov et al, Bull. Acad. Sci. USSR, Phys. Ser. 53, (1987) 1

24. S.T. Perkins et al, Lawrence Livennore National Laboratory, UCRL-50400 30, (1991)

25. I.M. Band et al, At. Data and Nucl. Data Tables 81, (2002) 1 